\title{
Minerals: An Untapped Remedy for Autoimmune Hypothyroidism?
}

Seyad Zulficar Ali Khan ${ }^{1,2}$, Rayan M. Lungba ${ }^{3}$, Uvie Ajibawo-Aganbi ${ }^{3}$, Swathi Veliginti ${ }^{3}$, Maria V. Perez Bastidas $^{3}$, Sania Saleem ${ }^{3}$, Ivan Cancarevic ${ }^{3}$

1. Research and Development, California Institute of Behavioral Neurosciences \& Psychology, Fairfield, USA 2. Primary Care \& Emergency, Ministry of Health Oman, Salalah, OMN 3. Internal Medicine, California Institute of Behavioral Neurosciences \& Psychology, Fairfield, USA

Corresponding author: Seyad Zulficar Ali Khan, zulfi223@gmail.com

\begin{abstract}
For decades, the focus of managing autoimmune hypothyroidism has been on thyroxine replacement. Correcting lab parameters such as thyroid stimulating hormone (TSH) has been a primary goal. The increasing prevalence of Hashimoto's thyroiditis (HT) continues to impact the quality of life in patients. We believe a holistic approach to this disease entity, considering its underlying complex etiopathogenesis, would benefit patients. Nutraceuticals are combinations of essential nutrients and are becoming a part of novel medical treatments despite the lack of regulation. This review aims to present a concise summary of recent developments regarding minerals such as zinc, selenium, magnesium, iron, and their potential clinical benefit as nutraceuticals in patients with HT. We have explored the potential benefits and associations of these minerals in HT and thyroid function. We reviewed relevant articles, metanalyses, and clinical trials in literature platforms such as PubMed, PubMed Central, and Google Scholar. Significant data found in the literature suggesting a potential health benefit of these minerals in HT though there were many studies to the contrary. Many trials demonstrated the restoration of residual symptoms, reversal of HTassociated thyroid morphological changes, and improvement in thyroid functions. Many of these trials lack statistical power due to the small sample sizes, and their external validity may be questionable due to unaccounted confounding factors. In our opinion, to support an evidence-based holistic clinical approach, further scientific evidence is needed. The association of these elements in thyroid function necessitates more large scale pragmatic trials to elucidate the benefits of nutraceuticals in HT.
\end{abstract}

Received 09/21/2020

Review began 09/30/2020 Review ended 10/12/2020 Published 10/17/2020

\section{() Copyright 2020}

Khan et al. This is an open access article distributed under the terms of the Creative Commons Attribution License CC-BY 4.0., which permits unrestricted use, distribution, and reproduction in any medium, provided the original author and source are credited.
Categories: Endocrinology/Diabetes/Metabolism, Internal Medicine, Allergy/Immunology Keywords: autoimmune thyroiditis, hashimoto's thyroiditis, hypothyroidism, nutraceuticals, zinc, selenium, magnesium, iodine, essential nutrients

\section{Introduction And Background}

Thyroid autoimmunity, commonly mentioned as autoimmune thyroid diseases (AITD) in literature, encompasses various disease entities that arise due to our immune system being intolerant to specific thyroid antigens. This immune intolerance can trigger a cascade of cellular and humoral immune reactions that could eventually damage the thyroid cellular infrastructure [1]. Immune-mediated insults often result in the dysfunction of this vital gland. As one of the most affected organs in the body by autoimmunity, researchers are puzzled by the low immune tolerance of this organ. Researchers have explored the possible interplay of environmental, nutritional, and genetic factors triggering this autoimmunity. The most prevalent disease entities resulting from autoimmune insult are Hashimoto's thyroiditis (HT) and Grave's disease (GD) [1]. HT manifests commonly as hypofunctioning of the gland and often reflects clinically with hypothyroidism features such as fatigue, constipation, irregular menses, cold intolerance, and weight gain, to name a few [1,2]. Meanwhile, GD manifests clinically with hyperthyroidism features such as palpitations, anxiety, menstrual dysfunction, heat intolerance, and weight loss [1,2].

HT is the most common cause of hypothyroidism in the developed world and areas with sufficient iodine repletion. In contrast, iodine deficiency still is the number one cause for hypothyroidism in regions where nutritional iodine deficiency exists [2]. HT is more commonly associated with anti-thyroid peroxidase antibody (TPO-Ab) and anti-thyroglobulin (TG) antibodies in serum resulting in lymphocyte infiltration, fibrosis in later stages. Its prevalence has been around five for every 1000 residents in the USA. HT more commonly affects females, and the average age of onset is 35-45 [3]. The prevalence is in an upswing bringing the spotlight on iodine overcorrection as a possible causal factor and necessitating further research on other influences in play. Analysis in Denmark indicated a 55\% co-occurrence rate of AITD in monozygotic twins compared to $3 \%$ in dizygotic. The study also suggested that around $79 \%$ of predisposition can be attributable to genetic factors and the remaining $21 \%$ to environmental, nutritional, and other influences [4].

In recent years, the role of nutritional deficiencies in the pathogenesis, disease perpetuation, and clinical manifestations of HT has drawn much attention. For instance, vitamin D deficiency and excessive iodine intake could potentially be the underlying cause of HT development $[5,6]$. There is evidence of a positive 
relationship between vitamin D and thyroid stimulating hormone (TSH), thyroxine (T4), and triiodothyronine (T3) levels in literature, yet unresolved questions remain. In one study, vitamin D was deficient in $76.7 \%$ of HT patients and $70 \%$ of Graves' disease patients compared to $20.0 \%$ in healthy people. However, its role in preventive and therapeutic aspects was not proven [5]. Iodine overcorrection is known to increase reactive oxygen species (ROS) in the thyroid gland. Accumulation of ROS can activate inflammatory pathways leading to thyrocyte apoptosis, thus playing a pivotal role in HT [6].

Apart from vitamin D and iodine excess, the crucial role of zinc, selenium, iron, and magnesium in thyroid pathophysiology has been a subject of interest. A small subset of 10 to $15 \%$ of the population with clinical manifestations of HT is, in fact, negative for antibody titers. In them, positive antibody titers only signified the symptom severity [7]. Further supporting the complexity of HT, studies reveal up to $10 \%$ of hypothyroid patients continue to experience symptoms even after receiving treatment to normalize serum TSH levels [8]. Possible explanations for this could be individual differences in sensitivity, unique variations in average values, and possibly an interplay of nutritional and environmental influences.

HT is presently managed with daily thyroxine supplementation to overcome the hypothyroid symptoms. Established protocols help clinicians decide on the appropriate initial dosing and then titer it to bring TSH within the standard reference range [3]. This review article will focus on how undetected nutritional deficiencies of zinc, selenium, iron, and magnesium can adversely impact patients with HT. Importantly we will search for any added benefit in addressing these deficiencies in HT patients in their overall quality of life, disease control, and lab parameters. We will explore the literature in PubMed, PubMed Central, and Google Scholar to enhance our knowledge of recent developments in this front. Our search will include both animal-model studies and human clinical trials to understand the associations better. We aim to determine if there is compelling evidence in the literature to incorporate screening for mineral deficiencies and supplement the needed minerals as part of comprehensive HT management. Figure 1 represents the various factors involved in the development of autoimmune thyroiditis [2,5-7].

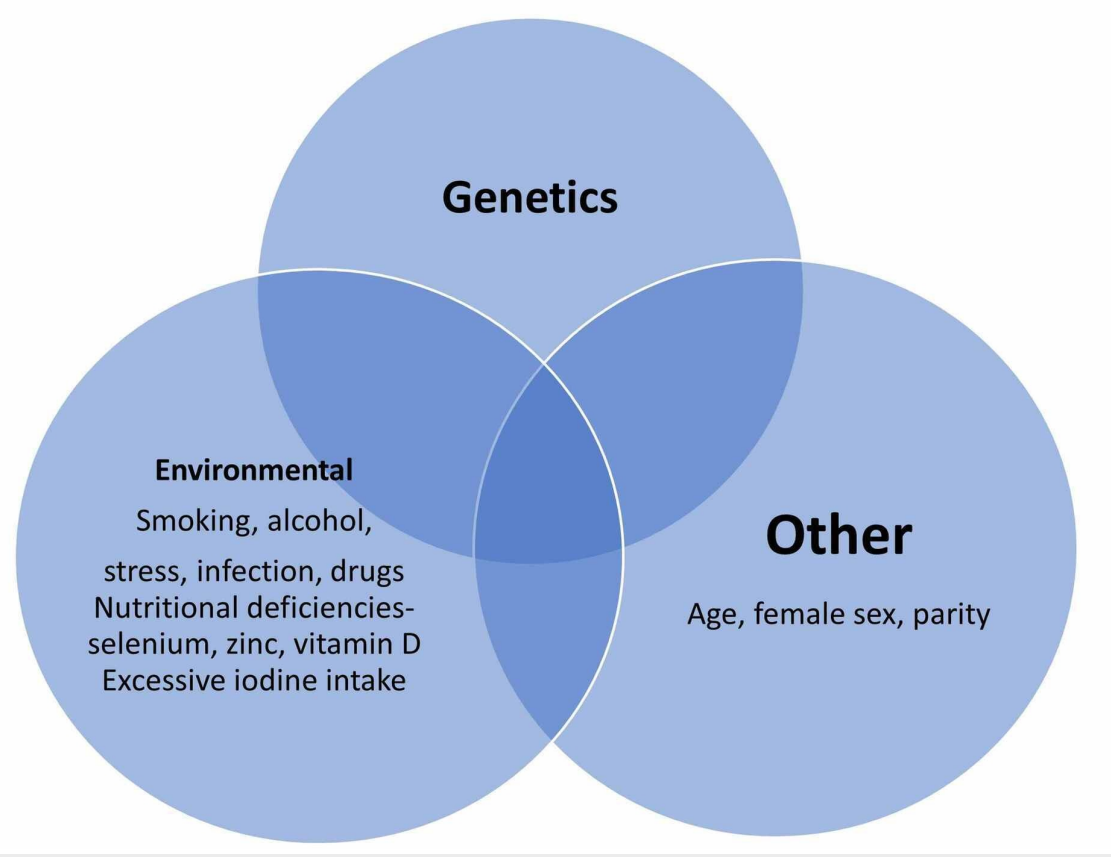

FIGURE 1: Factors influencing the development of Hashimoto's thyroiditis

\section{Review}

\section{Role of apoptosis in autoimmune thyroiditis}

Thyroid autoimmunity is an organ-specific immune disorder caused by aberrant immune regulation. Underlying pathogenesis can involve two distinct mechanisms in Grave's disease and Hashimoto's thyroiditis [9]. Researchers once thought that antibody-mediated cellular destruction and T-cell mediated cytotoxicity are the primary underlying mechanisms of HT. Recently, however, researchers have highlighted the crucial role of apoptosis in HT propagation [9]. Multiple in vitro studies have revealed high expression of pro-apoptotic molecules such as Fas, FasL, and Bax in thyroid follicular cells of HT patients, and reduced expression of the same in invading lymphocytes. This finding indicates an increased propensity for thyrocyte 
apoptosis and lymphocytes' survival. In contrast, studies done in GD show reduced expression of proapoptotic molecules and increased anti-apoptotic Bcl-2 expression in thyroid cells, which supports thyroid cell survival [10].

What triggers the cascade of apoptosis in thyroid glands of HT patients is yet poorly understood. A reasonable explanation would be an environmental, viral, nutritional deficiency, and oxidative stress on genetically susceptible patients [7]. For instance, essential microelements such as zinc, selenium, and magnesium all play a pivotal role in preserving and regulating cellular oxidative state [11-13]. Their role in antioxidant defense help prevents cell damage from oxidative stress [11-13]. Any imbalance in the redox state and defect in the antioxidant defense mechanism can trigger apoptosis and inflammation. Thus, the oxidative stress of thyroid cells is an essential factor in HT propagation [14].

\section{Oxidative stress as an underlying factor in Hashimoto's thyroiditis}

The significance of oxidative stress (OS) in the pathophysiology of HT has drawn much attention in recent years [14]. Reduction and oxidation (redox) are critical metabolic steps fundamental to cellular homeostasis and metabolism. They play a crucial role in cellular defense against invading infectious agents and signal transductions in immune processes [14]. Any imbalance in the redox state can result in elevated concentrations of oxidants such as reactive oxygen species (ROS), reactive nitrogen species (RNS), reactive sulfides (RS) in cells $[14,15]$. This increase in oxidants relative to reductants causes oxidative stress to cell components $[14,15]$. Increased OS destabilizes the cell membrane and other vital cell structures, causing alterations in DNA and its repair and degradation [15].

Antioxidants counteract oxidative stress. Some essential antioxidants are enzymatic such as catalases, glutathione peroxidases, superoxide dismutase, and thioredoxin system [16]. Some are non-enzymatic such as vitamins A, C, and E [16]. Recent studies have unraveled the potential role of ROS in the induction of autoimmunity and apoptosis $[16,17]$. ROS accumulation cause elevated oxidative stress, DNA instability, disruption of DNA repair, and degradation mechanisms [17]. This disruptive role of ROS results in the accumulation of single-stranded DNA (ssDNA) and double-stranded DNA (dsDNA) fragments in the cytoplasm [16,17]. Both ssDNA and dsDNA fragments are potent immune stimulators; they do this by inducing interferon (IFN) genes to increase the production of IFN [17]. Interferon, in turn, triggers autoreactivity and inflammation. The defective DNA repair mechanism is also a cause of innate immunity activation and inflammation [17].

A study highlighted the crucial role of OS in HT by demonstrating a significant negative correlation of antiTPO antibody with antioxidant status. They also found a similar negative correlation between antioxidant thiol and anti-TG antibody [14]. Baser et al., in a similar study, demonstrated a positive correlation of oxidative stress with the anti-TG antibody [18]. Ruggeri et al., in his research, suggested that the TPO antibody can be a marker and predictor of oxidative stress [19]. In an animal model study, ROS accumulation in thyroid cells promoted increased fragmentation of thyroglobulin, unmasking potential epitopes to innate immunity [20]. A similar study in mice revealed that increased oxidative stress caused increased susceptibility to iodine-induced apoptosis of thyrocytes [21]. Figure 2 highlights the progression to HT $[10,14,17]$.

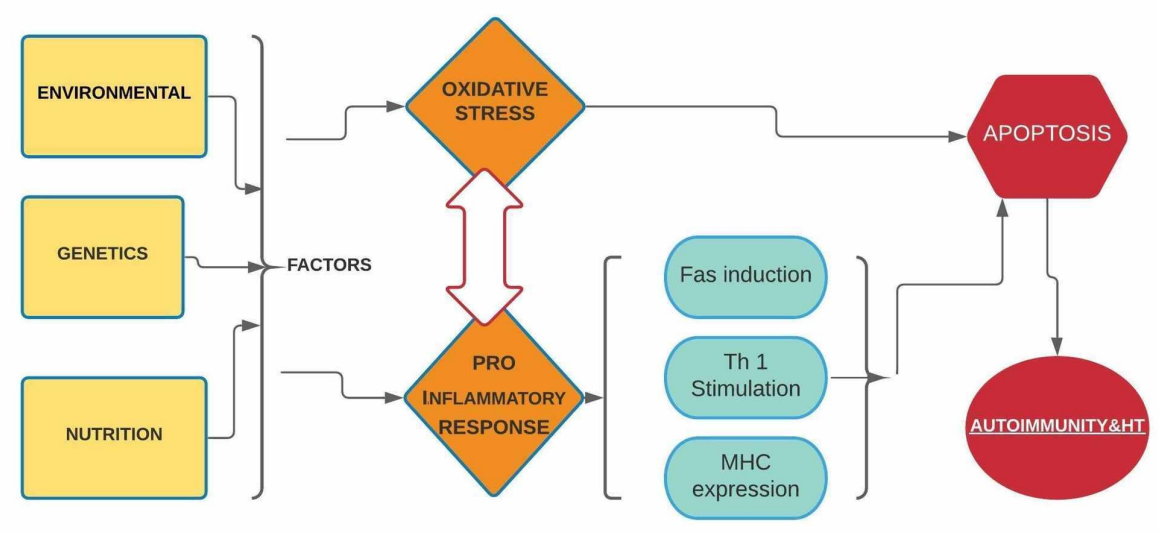

FIGURE 2: Pathway to autoimmunity \& Hashimoto's thyroiditis

MHC: major histocompatibility complex, Fas ligand: a transmembrane protein

Selenium, a crucial element in thyroid function 
Since its discovery, research has continued to unravel the vital role of selenium in the human body. Once side-lined as a carcinogen, discoveries of its diverse role in human health has perplexed researchers. It promotes cardiovascular wellbeing, endocrine regulation, and plays a more significant role in immunity. Selenium directly incorporates as the 21 st amino acid selenocysteine in various proteins. Interestingly the highest concentration of selenium is also found in the thyroid. Three out of 35 known selenoproteins play a crucial role in thyroid function. Some selenoproteins implicated in thyroid function are iodothyronine deiodinase-1 (DIO1), iodothyronine deiodinase-2 (DIO2), which are essential to maintaining intracellular and systemic active thyroid hormone (T3) levels [13]. Iodothyronine deiodinase-3 (DIO3), the other selenoprotein, helps to inactivate excess thyroxin [13,22]. Selenoprotein K (selenok) also plays a vital role in calcium influx in immune cells $[13,22]$. Some selenoproteins such as glutathione peroxidase, thioredoxin reductase, and sulphoxide reductase play a crucial role in immune regulation [22]. Glutathione peroxidase (GP)1, GPX3, and GPX4 catalyze peroxides and protect thyrocytes from oxidative damage [13]. It is envisaged that selenium is vital to maintain redox homeostasis, for efficient DNA damage repair, and to regulate proinflammatory cytokine synthesis. It also has a direct effect on innate immunity through the regulation of macrophage function [22].

Many studies have revealed an association of selenium deficiency with thyroid autoimmunity. A metanalysis published in 2016 explored the benefit of selenium supplementation in HT. They concluded that selenium supplementation could significantly reduce thyroid autoantibodies in patients with HT. They compared both patients on thyroxin substitution and patients not on thyroxine substitution. Their study revealed the beneficial effects of three-month selenium supplementation in patients not on thyroxine therapy [23]. Pirola et al. conducted a prospective clinical trial to evaluate the benefit of $83 \mathrm{mcg}$ once-daily selenium supplementation for four months in patients with HT. The trial demonstrated the restoration of euthyroidism in $31.3 \%$ of responders amongst the selenium group compared to only $3.1 \%$ in controls [24]. In a separate study, Pirola et al. demonstrated the TSH lowering effect of short-course selenium supplementation. His research also suggested that the TSH lowering effect lasted six months after the withdrawal of selenium supplements [25]. In an earlier study conducted by Gartner and co-workers, they showed a decrease of $40 \%$ in mean TPO antibody titers after supplementing selenium. They backed their study with ultrasound imaging of the thyroid gland, which showed a reduced echogenicity in the selenium group compared to the controls, indicating a beneficial effect in lowering thyroid inflammation in patients with HT [26].

A prospective randomized clinical trial conducted in Italy in 2017 enrolled 168 patients with HT to evaluate the added benefit of Myo-inositol and selenium co-supplementation. After six-months of supplements, improvements in subjective symptoms and thyroid function parameters were assessed. The study concluded that $83 \mathrm{mcg}$ of selenium supplementation with $600 \mathrm{mg}$ of inositol daily significantly improved their symptoms and restored the euthyroid state [27].

Pregnancy increases the basal metabolic rate, and the demand for thyroxine rises during pregnancy. A study named SERENA demonstrated the benefit of selenium as a supplement in pregnant women with HT. The study enrolled 45 pregnant women with AITD and started daily selenium supplementation of $83 \mathrm{mcg}$, and assessments at the first-trimester end, at term, and postpartum were done. Although the study failed to show any added benefit in quality of life and pregnancy-related complications, it revealed significant reductions in TG antibody and TPO antibody titers $(\mathrm{P}<0.01)$ [28]. A study in the UK explored the benefit of low-dose selenium supplements in thyroid antibody titers of pregnant women from low iodine intake communities. The study classified patients based on their thyroid antibody status. It concluded that significant TSH reductions only in the TPO-Ab group, and TPO-Ab titers did not change significantly. Considering that study did not recruit patients with known thyroid diseases, it is unclear how patients with HT and on thyroxine supplements would have responded to low-dose selenium supplements. This study also raises the possibility of iodine status as a potential confounding factor in studies related to thyroid and selenium intake [29].

Our search retrieved a total of 35 relevant clinical trials and three metanalyses. Two studies assessed the cosupplementation of selenium and myoinositol in AITD [27]. One study assessed selenium and vitamin C cosupplementation in AITD, and three other studies assessed selenium supplementation in thyroid autoimmunity of pregnant women $[28,29]$. There is also one study investigating selenium and vitamin D cosupplementation in AITD. There are 11 other clinical trials related to the assessment of selenium supplementation on thyroid function in healthy people. There are 16 clinical trials directly assessing the effect of selenium supplementation in AITD. Many of these trials evaluated the effect of selenium supplementation on TPO antibodies. Many studies have found a significant decrease in TPO-Ab after three to 12 months of selenium supplementation [30,31]. However, a few studies did not find a substantial outcome in antibody titers [32]. One study assessed the effect on the quality of life though it failed to prove any significant outcome [33].

In our assessment, the above studies, in general, need to be seen in the light of limitations such as small sample sizes and their potential for confounding factors like age, gender, thyroid status, and other possible comorbidities. Though these studies are promising, in the future, we suggest extensive population-based pragmatic studies that would help delineate the beneficial role of selenium supplementation in clinical practice. One such community-based, large pragmatic study named CATALYST (chronic autoimmune 
thyroiditis quality of life selenium trial) specifically looks into the outcome in quality of life in AITD, and results are awaited [34]. Table 1 summarizes some of the important studies related to selenium supplementation in AITD.

\begin{tabular}{|c|c|c|c|c|}
\hline $\begin{array}{l}\text { Author \& } \\
\text { Year }\end{array}$ & $\begin{array}{l}\text { Aim of selenium } \\
\text { supplementation }\end{array}$ & $\begin{array}{l}\text { Study type \& } \\
\text { (n) }\end{array}$ & Supplementation method & Results \\
\hline $\begin{array}{l}\text { Pirola et } \\
\text { al. } 2016 \\
{[24]}\end{array}$ & $\begin{array}{l}\text { Effect on subclinical } \\
\text { hypothyroidism in } \\
\text { AITD }\end{array}$ & RCT, n-196 & 83mcg-OD for 4-Months & $17.2 \%$ achieved euthyroidism. \\
\hline $\begin{array}{l}\text { Pirola et } \\
\text { al. } 2019 \\
{[25]}\end{array}$ & $\begin{array}{l}\text { Effect on TSH, } \\
\text { autoimmune } \\
\text { markers in AITD }\end{array}$ & $\begin{array}{l}\text { Prospective } \\
\text { observational. } \\
n-50\end{array}$ & $83 \mathrm{mcg}-\mathrm{OD}$ for 4 Months & $\begin{array}{l}48.9 \% \text { of responders achieved euthyroidism. } \\
\text { No significant change in TPOAb \& } \\
\text { chemokines. }\end{array}$ \\
\hline $\begin{array}{l}\text { Gartner et } \\
\text { al. } 2002 \\
{[26]}\end{array}$ & $\begin{array}{l}\text { Effect on antibody } \\
\text { titers, thyroid } \\
\text { function, volume \& } \\
\text { QoL in AITD. }\end{array}$ & RCT, n-70 & 200mcg-OD for 3-Months & $\begin{array}{l}40 \% \text { reduction of TPOAb in the selenium } \\
\text { group compared to } 10 \% \text { in placebo. No } \\
\text { significant changes in TgAb, T4, T3. USS } \\
\text { improved in nine patients. }\end{array}$ \\
\hline $\begin{array}{l}\text { Mantovani } \\
\text { et al. } 2019 \\
{[28]}\end{array}$ & $\begin{array}{l}\text { Effect on AITD } \\
\text { among pregnant } \\
\text { women. }\end{array}$ & RCT, n-45 & $\begin{array}{l}\text { 83mcg-OD Till 6-Months } \\
\text { postpartum. }\end{array}$ & $\begin{array}{l}\text { Significant reductions in TPOAb }(P<0.01) \text {, } \\
\operatorname{TgAb}(P<0.01) \text {. No changes in } Q o L .\end{array}$ \\
\hline $\begin{array}{l}\text { Kyrgios et } \\
\text { al. } 2018 \\
{[31]}\end{array}$ & $\begin{array}{l}\text { Effect of high dose } \\
\text { selenium on AITD }\end{array}$ & RCT, n-71 & 200mcg- OD for 6-Months & $\begin{array}{l}\text { Reductions in TgAb }(P=0.021) \text {. TPOAb not } \\
\text { significant }(P=0.219) \text {. USS no changes. }\end{array}$ \\
\hline $\begin{array}{l}\text { Bonfig et } \\
\text { al. } 2010 \\
{[32]}\end{array}$ & $\begin{array}{l}\text { Effect on antibody } \\
\text { titers }\end{array}$ & RCT, n-49 & $\begin{array}{l}\text { Group A-Thyroxine alone.Group } \\
\text { B-Thyroxine + selenium } \\
\text { 100mcg.Group c-Thyroxine + } \\
\text { selenium } 200 \mathrm{mcg}\end{array}$ & $\begin{array}{l}\text { Reductions in } T g A b \text { in Group } A(P=0.03) \& \\
\text { Group } C(P=0.01) \text {. Group } B \text {, not significant } \\
(P=0.06) \text {. No significant changes in TPOAb in } \\
\text { all groups. }\end{array}$ \\
\hline $\begin{array}{l}\text { Eskes et } \\
\text { al. } 2013 \\
\text { [33] }\end{array}$ & $\begin{array}{l}\text { Effect on euthyroid } \\
\text { patients with } \\
\text { positive TPOAb. }\end{array}$ & RCT, n-61 & 200mcg- OD for 6-Months & No effect on QoL and TPOAb titers. \\
\hline
\end{tabular}

\section{TABLE 1: Influence of selenium in AITD.}

OD: once daily, AITD: autoimmune thyroid disease, QoL: quality of life, RCT: randomized control trials, TPOAb: anti-thyroid peroxidase antibody, TgAb: anti-thyroglobulin antibodies, T4: thyroxine, T3: triiodothyronine

\section{Zinc as an antioxidant}

We cannot overstate the vital role of zinc in the human body. It is a potent antioxidant, anti-inflammatory, and immune regulating element [35]. Its deficiency increases autoimmune susceptibility in general [36].

A randomized control trial conducted in 2015 explored the potential benefit of both selenium and zinc either alone or in combination with thyroid function. The study concluded a beneficial outcome with either zinc alone or in combination with selenium in thyroid function [37]. Another study aimed to evaluate the role of zinc supplementation in hypothyroidism in patients with Down's syndrome. The study demonstrated improved thyroid parameters, specifically TSH, after supplementing zinc for six months [38]. In an earlier study conducted in 2009, researchers explored the benefit of zinc supplementation in male and female patients with goiters. The study reported a beneficial outcome in thyroid function after six months of zinc supplementation [36]. Though the above studies indicate a potential benefit in thyroid status with zinc supplementation, their role in autoimmune thyroiditis is yet to be explored and established. Previous research on zinc's role in multiple autoimmune diseases suggests a potential benefit in preserving immune tolerance [39].

\section{Magnesium for iodine uptake}

To date, limited data available in the literature on the association of magnesium in thyroid function. However, studies on the benefit of magnesium supplementation in the body's overall oxidative status and inflammation have been published $[11,40]$. Thyroid hormones play a vital role in mitochondrial function. Adenosine triphosphate (ATP) production in mitochondria is essential to maintain energy-dependent cell reactions, including iodine uptake through sodium-iodine symporters, a crucial step in thyroid hormone 
production. Magnesium is an essential element in oxidative phosphorylation as part of complex V [40]. A study concluded that elevated thyroid vasculature associated with HT's inflammation correlated negatively with serum magnesium levels [41]. Another similar study analyzed the outcome of co-supplementation of magnesium, selenium, and coenzyme Q10 in patients with benign thyroid conditions. After two to four years of supplementation, they found a significant drop in antibody titers and normalization of thyroid morphology as evidenced by high-resolution USS [42]. This study certainly has limitations considering the small sample size and potential for biases and confounding factors.

HT encompasses a diverse range of symptoms, which can even present in patients with unremarkable lab and physical parameters. Symptoms such a chronic fatigue, irritability, low concentration, anxiety, nervousness, and even unexplained miscarriages pose a dilemma to medical professionals. Commonly these symptoms are categorized as psychosomatic or medically unexplained symptoms (MUS) [43]. Not recognizing or failing to address these symptoms can adversely affect the quality of life in these patients. There is ample evidence in the literature of co-existing physical and psychosocial stressors in HT and hypothyroidism [43]. A study found that hypothyroidism and other physical/psychosocial stressors can increase magnesium demand. This study included 166 euthyroid and 108 hypothyroid patients. It assessed the stress levels by a scoring system before and after magnesium supplementation in three and six months. They found that $90 \%$ of patients had their psychosomatic symptoms resolved after magnesium supplementation. A significant portion of patients also reported improved quality of life [44]. Though these studies may not hold sufficient evidence to suggest magnesium's benefit in hypothyroidism scientifically, they highlight the need for future studies in this direction.

\section{Iron for optimal thyroid functioning}

Thyroid peroxidase (TPO), a hemoprotein in thyrocytes, has a significant role in thyroid hormone synthesis. It is crucial for iodine oxidation and integration into thyroglobulin. There is evidence from multiple animal model studies that TPO activity diminishes under iron deficiency. These studies imply the need for iron sufficiency to maintain optimum thyroid function [45]. Further, Hu et al., in his studies in rats, demonstrated iron deficiency alone without anemia can lead to suboptimal thyroxine production [46].

In his study, Yu et al. concluded that iron deficiency could be an independent risk factor for hypothyroxinaemia in pregnant and non-pregnant mothers [47]. In his study in pregnant women, Zimmermann et al. demonstrated that reduced iron stores could negatively correlate with the TSH while showing a positive relationship between iron stores and thyroid hormones. He concluded that optimal iron stores could achieve a better thyroid function [48].

A large cohort study demonstrated the significance of thyroid hormone in maintaining hemoglobin and hematocrit levels. The study reveals TSH correlated negatively with iron stores, transferrin saturation [49]. Thyroid hormones augment erythropoietin gene expression in the kidney and induce hematopoiesis. Hypothyroid status reduces basal metabolic rate resulting in decreased demand for oxygen and erythropoiesis. So, reduced thyroid hormones do lower erythropoiesis, further signifying the importance of sufficient iron stores [49].

Further, AITD is associated with multiple other autoimmune diseases that could be an additional causal factor for anemia [50]. These include pernicious anemia, celiac disease, autoimmune hemolytic disease, and rheumatism, which causes anemia of chronic diseases. In women, heavy menstrual bleeding is a frequent manifestation of hypothyroid status and often results in iron deficiency [50]. Data on the prevalence of anemia in subclinical thyroid dysfunction are inconsistent in the literature. Studies have revealed the incidence of anemia among hypothyroid patients at 57\% [50]. The importance of maintaining iron sufficiency in hypothyroid patients can be attributed to its role in thyroid hormone synthesis and due to the increased susceptibility of developing anemia in these patients. Further large-scale cohort studies are needed to determine if any causal relationship exists between iron deficiency and AITD. Figure 3 demonstrates the influences of these minerals in thyroid function $[11,22,35,40,45]$. 


\section{Cureus}

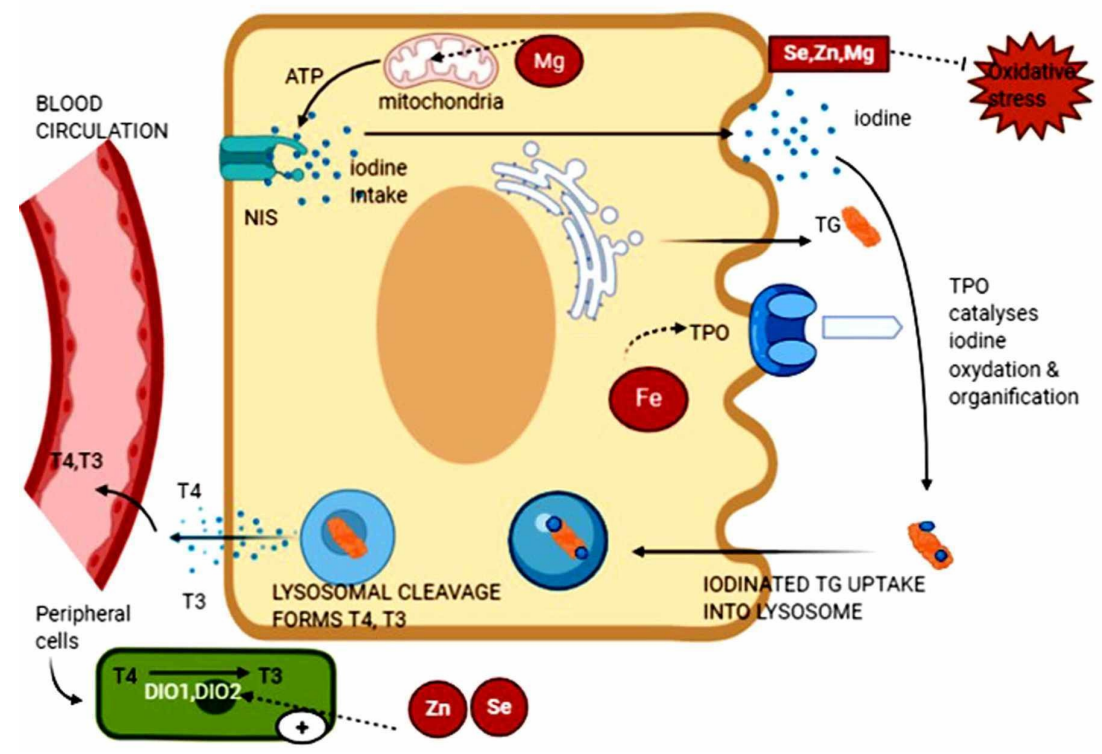

FIGURE 3: Overview of the influence of minerals in thyroid physiology

NIS: sodium iodide symporter, TPO: thyroid peroxidase enzyme, DIO1,2: iodothyronine deiodinase 1,2, ATP: adenosine triphosphate, TG: thyroglobulin, T4: thyroxine, T3: triiodothyronine

\section{Limitations in our review}

Our review has some limitations; we did not carry out quality assessments on the chosen studies and articles. Some of the researches related to etiopathogenesis are based on animal models. Most of the clinical trials were with inadequate statistical power and non-pragmatic. They may not apply to different populations and settings. The decision to supplement nutraceuticals should not be based solely on hypothetical health benefits. Dosing requirements can vary based on individual diet practices and geographical areas due to variations in soil nutrients and agricultural practices. Potential for harm due to high doses, especially in susceptible populations such as children and the elderly, also needs to be considered.

\section{Conclusions}

In this review, we have attempted to present a concise summary of the potential benefits of micronutrients zinc, selenium, magnesium, and iron on overall thyroid function and in HT. Oxidative stress and apoptosis play a significant role in HT's etiopathogenesis. Oxidative DNA damage and cell apoptosis are initial events triggering autoimmunity in the thyroid. Preserving oxidative status and optimizing nutrients can help improve thyroid function. It is evident from many studies that selenium and zinc play an essential role in preserving thyrocytes' oxidative status and prevent apoptosis and cell damage. Magnesium has a critical role in the functioning of sodium iodide symporter, which helps maintain a steady iodine supply for thyroid hormone synthesis. Iron deficiency is strongly associated with hypothyroid status, and sufficient iron stores are vital for the thyroid function. Large scale clinical trials are needed in the future to consider incorporating supplements as a novel evidence-based remedy in clinical practice.

\section{Additional Information}

\section{Disclosures}

Conflicts of interest: In compliance with the ICMJE uniform disclosure form, all authors declare the following: Payment/services info: All authors have declared that no financial support was received from any organization for the submitted work. Financial relationships: All authors have declared that they have no financial relationships at present or within the previous three years with any organizations that might have an interest in the submitted work. Other relationships: All authors have declared that there are no other relationships or activities that could appear to have influenced the submitted work.

\section{References}

1. Antonelli A, Ferrari SM, Corrado A, et al.: Autoimmune thyroid disorders. Autoimmun Rev. 2015, 14:174180. 10.1016/j.autrev.2014.10.016

2. Chiovato L, Magri F, Carlé A: Hypothyroidism in context: where we have been and where we are going . Adv Ther. 2019, 36:47-58. 10.1007/s12325-019-01080-8

3. Vanderpump MP: The epidemiology of thyroid disease . Br Med Bull. 2011, 99:39-51. 10.1093/bmb/ldr030 
4. Brix TH, Hegedüs L, Gardas A, et al.: Monozygotic twin pairs are discordant for Hashimoto's thyroiditis share a high proportion of thyroid peroxidase autoantibodies to the immunodominant region A. Further evidence for genetic transmission of epitopic “fingerprints.”. Autoimmunity. 2011, 44:188-94. 10.3109/08916934.2010.518575

5. Bakr HG, Meawed TE: The relevance of $25(\mathrm{OH})$ vitamin D deficiency on Hashimoto's thyroiditis . Egypt J Immunol. 2017, 24:53-62.

6. Liu J, Mao C, Dong L, et al.: Excessive iodine promotes pyroptosis of thyroid follicular epithelial cells in Hashimoto's thyroiditis through the ROS-NF-KB-NLRP3 pathway. Front Endocrinol Lausanne. 2019, 10:778. 10.3389/fendo.2019.00778

7. Mincer DL, Jialal I: Hashimoto Thyroiditis. StatPearls, Treasure Island; 2020.

8. Jonklaas J: Persistent hypothyroid symptoms in a patient with a normal thyroid-stimulating hormone level . Curr Opin Endocrinol Diabetes Obes. 2017, 24:356-363. 10.1097/med.0000000000000355

9. Chen S, Fazle Akbar SM, Zhen Z, et al.: Analysis of the expression of Fas, FasL, and Bcl-2 in the pathogenesis of autoimmune thyroid disorders. Cell Mol Immunol. 2004, 1:224-228.

10. Zhang Y, Ji Q, Zhang W: Expression of apoptosis-related proteins in Hashimoto's thyroiditis and its pathological significance [Article in Chinese]. Zhonghua Yi Xue Za Zhi. 2001, 81:432-4.

11. Zheltova AA, Kharitonova MV, Iezhitsa IN, et al.: Magnesium deficiency and oxidative stress: an update . Biomedicine Taipei. 2016, 6:20. 10.7603/s40681-016-0020-6

12. Blewett HJ, Taylor CG: Dietary zinc deficiency in rodents: effects on T-cell development, maturation, and phenotypes. Nutrients. 2012, 4:449-66. 10.3390/nu4060449

13. Avery JC, Hoffmann PR: Selenium, selenoproteins, and immunity. Nutrients. 2018, 10:1203. 10.3390/nu10091203

14. Ates I, Yilmaz FM, Altay M, et al.: The relationship between oxidative stress and autoimmunity in Hashimoto's thyroiditis. Eur J Endocrinol. 2015, 173:791-9. 10.1530/EJE-15-0617

15. Theofilopoulos AN, Kono DH, Baccala R: The multiple pathways to autoimmunity. Nat Immunol. 2017, 18:716-724. 10.1038/ni.3731

16. Birben E, Sahiner UM, Sackesen C, et al.: Oxidative stress and antioxidant defense. World Allergy Organ J. 2012, 5:9-19. 10.1097/WOX.0b013e3182439613

17. Brzostek-Racine S, Gordon C, Van Scoy S, et al.: The DNA damage response induces IFN . J Immunol. 2011, 187:5336-5345. 10.4049/jimmunol.1100040

18. Baser H, Can U, Baser S, et al.: Assessment of oxidative status and its association with thyroid autoantibodies in patients with euthyroid autoimmune thyroiditis. Endocrine. 2015, 48:916-923. 10.1007/s12020-014-0399-3

19. Ruggeri RM, Vicchio TM, Cristani M, et al.: Oxidative stress and advanced glycation end products in Hashimoto's thyroiditis. Thyroid. 2016, 26:504-511. 10.1089/thy.2015.0592

20. Duthoit C, Estienne V, Giraud A, et al.: Hydrogen peroxide-induced production of a $40 \mathrm{kDa}$ immunoreactive thyroglobulin fragment in human thyroid cells: the onset of thyroid autoimmunity?. Biochem J. 2001, 360:557-562. 10.1042/0264-6021:3600557

21. Kolypetri P, Carayanniotis G: Apoptosis of NOD.H2h4 thyrocytes by low concentrations of iodine are associated with impaired control of oxidative stress. Thyroid. 2014, 24:1170-1178. 10.1089/thy.2013.0676

22. Roman M, Jitaru P, Barbante C: Selenium biochemistry and its role for human health. Metallomics. 2014, 6:25-54. 10.1039/c3mt00185g

23. Wichman J, Winther KH, Bonnema SJ, et al.: Selenium supplementation significantly reduces thyroid autoantibody levels in patients with chronic autoimmune thyroiditis: a systematic review and metaanalysis. Thyroid. 2016, 26:1681-1692.

24. Pirola I, Gandossi E, Agosti B, et al.: Selenium supplementation could restore euthyroidism in subclinical hypothyroid patients with autoimmune thyroiditis. Endokrynol Pol. 2016, 67:567-571. 10.5603/EP.2016.0064

25. Pirola I, Rotondi M, Cristiano A, et al.: Selenium supplementation in patients with subclinical hypothyroidism affected by autoimmune thyroiditis: results of the SETI study. Endocrinol Diabetes Nutr. 2020, 67:28-35. 10.1016/j.endinu.2019.03.018

26. Gärtner R, Gasnier BC, Dietrich JW, et al.: Selenium supplementation in patients with autoimmune thyroiditis decreases thyroid peroxidase antibodies concentrations. J Clin Endocrinol Metab. 2002, 87:16871691. 10.1210/jcem.87.4.8421

27. Nordio M, Basciani S: Myo-inositol plus selenium supplementation restores the euthyroid state in Hashimoto's patients with subclinical hypothyroidism. Eur Rev Med Pharmacol Sci. 2017, 21:51-59.

28. Mantovani G, Isidori AM, Moretti C, et al.: Selenium supplementation in the management of thyroid autoimmunity during pregnancy: results of the "SERENA study," a randomized, double-blind, placebocontrolled trial. Endocrine. 2019, 66:542-550. 10.1007/s12020-019-01958-1

29. Mao J, Pop VI, Bath SC, et al.: Effect of low-dose selenium on thyroid autoimmunity and thyroid function in UK pregnant women with mild-to-moderate iodine deficiency. Eur J Nutr. 2016, 55:55-61. 10.1007/s00394014-0822-9

30. Balázs C: The effect of selenium therapy on autoimmune thyroiditis . Orv Hetil. 2008, 149:1227-32. 10.1556/OH.2008.28408

31. Kyrgios I, Giza S, Kotanidou EP, et al.: 1-selenomethionine supplementation in children and adolescents with autoimmune thyroiditis: a randomized, double-blind placebo-controlled clinical trial. J Clin Pharm Ther. 2019, 44:102-108. 10.1111/jcpt.12765

32. Bonfig W, Gärtner R, Schmidt H: Selenium supplementation does not decrease thyroid peroxidase antibody concentration in children and adolescents with autoimmune thyroiditis. Sci World J. 2010, 10:990-6. 10.1100/tsw.2010.91

33. Eskes SA, Endert E, Fliers E, et al.: Selenite supplementation in euthyroid subjects with thyroid peroxidase antibodies. Clin Endocrinol Oxf. 2014, 80:444-51. 10.1111/cen.12284

34. Winther KH, Watt T, Bjørner JB, et al.: The chronic autoimmune thyroiditis quality of life selenium trial (CATALYST): study protocol for a randomized controlled trial. Trials. 2014, 15:115. 10.1186/1745-6215-15- 
115

35. Choi S, Liu X, Pan Z: Zinc deficiency and cellular oxidative stress: prognostic implications in cardiovascular diseases. Acta Pharmacol Sin. 2018, 39:1120-1132. 10.1038/aps.2018.25

36. Kandhro GA, Kazi TG, Afridi HI, et al.: Effect of zinc supplementation on the zinc level in serum and urine and their relation to thyroid hormone profile in male and female goitrous patients. Clin Nutr. 2009, 28:162168. 10.1016/j.clnu.2009.01.015

37. Mahmoodianfard S, Vafa M, Golgiri F, et al.: Effects of zinc and selenium supplementation on thyroid function in overweight and obese hypothyroid female patients: a randomized, double-blind controlled trial. J Am Coll Nutr. 2015, 34:391-399. 10.1080/07315724.2014.926161

38. Bucci I, Napolitano G, Giuliani C, et al.: Zinc sulfate supplementation improves thyroid function in hypozincemic Down children. Biol Trace Elem Res. 1999, 67:257-268. 10.1007/BF02784425

39. Sanna A, Firinu D, Zavattari P, et al.: Zinc status, and autoimmunity: a systematic review and meta-analysis . Nutrients. 2018, 10:68. 10.3390/nu10010068

40. Jamilian M, Mirhosseini N, Eslahi M, et al.: The effects of magnesium-zinc-calcium-vitamin D cosupplementation on biomarkers of inflammation, oxidative stress, and pregnancy outcomes in gestational diabetes. BMC Pregnancy Childbirth. 2019, 19:107. 10.1186/s12884-019-2258-y

41. Moncayo R, Moncayo $\mathrm{H}$ : The WOMED model of benign thyroid disease: acquired magnesium deficiency due to physical and psychological stressors relates to dysfunction of oxidative phosphorylation. BBA Clin. 2014, 3:44-64. 10.1016/j.bbacli.2014.11.002

42. Moncayo R, Moncayo H: Proof of concept of the WOMED model of benign thyroid disease: restitution of thyroid morphology after correction of physical and psychological stressors and magnesium supplementation. BBA Clin. 2014, 3:113-22. 10.1016/j.bbacli.2014.12.005

43. Moncayo R, Moncayo H: Exploring the aspect of psychosomatics in hypothyroidism: the WOMED model of body-mind interactions based on musculoskeletal changes, psychological stressors, and low levels of magnesium. Woman. 2014, 1:1-11. 10.1016/j.woman.2014.02.001

44. Ott J, Promberger R, Kober F, et al.: Hashimoto's thyroiditis affects symptom load and quality of life unrelated to hypothyroidism: a prospective case-control study in women undergoing thyroidectomy for benign goiter. Thyroid. 2011, 161-167. 10.1089/thy.2010.0191

45. Hess SY, Zimmermann MB, Arnold M, et al.: Iron deficiency anemia reduces thyroid peroxidase activity in rats. J Nutr. 2002, 132:1951-1955. 10.1093/jn/132.7.1951

46. Hu X, Xiaochun T, Hongzhi Z, et al.: Iron deficiency without anemia causes maternal hypothyroxinaemia in pregnant rats. Nutr Res. 2014, 34:604-612. 10.1016/j.nutres.2014.06.007

47. Yu X, Zhongyan S, Li C, et al.: Iron deficiency, an independent risk factor for isolated hypothyroxinemia in pregnant and non-pregnant women of childbearing age in China. J Clin Endocrinol Metab. 2015, 100:15941601. 10.1210/jc.2014-3887

48. Zimmermann MB, Hans B, Richard FH, et al.: Iron deficiency predicts poor maternal thyroid status during pregnancy. J Clin Endocrinol Metab. 2007, 92:3436-3440. 10.1210/jc.2007-1082

49. Bremner AP, Feddema P, Joske DJ, et al.: Significant association between thyroid hormones and erythrocyte indices in euthyroid subjects. Clin Endocrinol Oxf. 2012, 76:304-311. 10.1111/j.1365-2265.2011.04228.x

50. Szczepanek-Parulska E, Hernik A, Ruchała M: Anaemia in thyroid diseases. Pol Arch Intern Med. 2017, 127:352-360. 10.20452/pamw.3985 